\title{
Joint Exposure to Chemical and Nonchemical Neurodevelopmental Stressors in U.S. Women of Reproductive Age in NHANES
}

\author{
Amanda M. Evans ${ }^{1, *}$, Glenn E. Rice ${ }^{2}$, Linda K. Teuschler ${ }^{3}$ and J. Michael Wright ${ }^{2}$
}

1 Oak Ridge Institute for Science and Education (ORISE), U.S. Environmental Protection Agency (EPA), Office of Research and Development, National Center for Environmental Assessment, Cincinnati, OH 45268, USA

2 U.S. EPA, Office of Research and Development, National Center for Environmental Assessment, Cincinnati, OH 45268, USA; E-Mails: Rice.Glenn@epa.gov (G.R.); Wright.Michael@epa.gov (M.W.)

3 Linda Teuschler and Associates, St. Petersburg, FL 33707, USA; E-Mail: lindateuschler@gmail.com

* Author to whom correspondence should be addressed; E-Mail: Evans.AmandaM@epa.gov; Tel.: +1-513-569-7458; Fax: +1-513-487-2540.

Received: 17 March 2014; in revised form: 5 April 2014 / Accepted: 10 April 2014 /

Published: 22 April 2014

Abstract: Lead $(\mathrm{Pb})$ and methyl mercury $(\mathrm{MeHg})$ are well established neurodevelopmental toxicants (NDTs), but joint exposure to chemical and nonchemical (e.g., maternal stress) stressors has rarely been considered. We characterized exposure to $\mathrm{Pb}, \mathrm{MeHg}$ and a measure of physiological dysregulation associated with chronic stress and examined race/ethnicity as a predictor of joint NDT exposure. Using data from the 2003-2004 NHANES, potential chronic stress exposure was estimated using allostatic load (AL), a quantitative measure of physiological dysregulation. A Hazard Index was calculated for joint exposure to $\mathrm{Pb}$ and $\mathrm{MeHg}\left(\mathrm{HI}_{\mathrm{NDT}}\right)$. Logistic regression was used to assess the relationship between an indicator of elevated joint NDT exposures $\left(\mathrm{HI}_{\mathrm{NDT}}>1\right)$ and race/ethnicity. The multivariate model was stratified by AL groups to examine effect measure modification. African American (adjusted odds ratio [OR] [95\% confidence interval $]=2.2[1.4,3.3])$ and Mexican American $(1.4[0.7,2.6])$ women were more likely to have an $\mathrm{HI}_{\mathrm{NDT}}>1$ compared to Caucasian women. Chronic stress was identified as an effect measure modifier with the largest ORs among women with high AL scores (African Americans $=4.3[2.0,9.5]$; Mexican Americans = 4.2 [1.3, 14.1]). Chronic stress was found to modify the association between elevated joint NDT exposure and race/ethnicity, 
highlighting the importance of evaluating chemical and nonchemical stressor exposures leading to a common endpoint.

Keywords: allostatic load (AL); chronic stress; cumulative exposure assessment; cumulative risk assessment (CRA); hazard index (HI); nonchemical stressors; neurodevelopment; physiological dysregulation; susceptibility; vulnerability

\section{Introduction}

In utero exposures to neurodevelopmental stressors, both individually and in combination, are public health concerns [1,2]. Although lead $(\mathrm{Pb})$ and methyl mercury $(\mathrm{MeHg})$ are known human neurodevelopmental toxicants (NDTs) at low exposure levels [3,4], few studies have examined their co-exposure [5] or joint toxicity [6]. Maternal prenatal stress also may adversely affect neurodevelopment [7].

Certain populations may be at increased risk of elevated exposure to NDTs and chronic stress. For example, African Americans have exhibited elevated blood $\mathrm{Pb}$ levels [8] and elevated chronic stress exposure [9]. Limited human studies suggest interactions between $\mathrm{Pb}$ and stress [10]. Although none of these epidemiological studies focused on reproductive-aged or pregnant women, toxicological data show that joint prenatal exposure to stress and $\mathrm{Pb}$ can result in an increased risk of adverse neurodevelopmental outcomes not observed in animals exposed to either stressor independently [11]. It has been hypothesized that the neurodevelopmental effects observed in toxicological studies examining joint $\mathrm{Pb}$ and stress exposure may be a result of shared toxicity targets, including the hypothalamic-pituitary-adrenal (HPA) axis and the dopamine and glutamatergic systems [11,12]. We identified no studies that examined joint exposure to $\mathrm{Pb}, \mathrm{MeHg}$, and stress in reproductive-aged women or any other populations.

Although blood levels of $\mathrm{Pb}$ and $\mathrm{MeHg}$ (half-lives of approximately 1 and 2 months, respectively) are reliable surrogates of recent exposures [13,14], quantifying chronic stress exposure is more challenging. Stress is a consequence of perceiving an "exposure" (e.g., violence, poverty) as more than one can handle (i.e., "stressful") [15]. Humans respond to stressful exposures via measurable physiological changes mediated by the HPA axis [16]. Chronic stress can result in physiological dysregulation of the stress response including both primary mediators (i.e., cortisol and the catecholamines) and secondary outcome measures (e.g., blood pressure, C-reactive protein), which can be quantitatively estimated via allostatic load (AL) $[16,17]$. AL has been defined as the number of physiological measures (i.e., primary mediators and secondary outcomes) across multiple systems (e.g., cardiovascular, metabolic, immune), responding outside of the normal range [16].

We examine race/ethnicity as a predictor of joint neurodevelopmental toxicant (NDT) exposure to $\mathrm{Pb}$ and $\mathrm{MeHg}$ in a nationally-representative sample of nonpregnant, reproductive-aged, women. Additionally, AL is used as an indicator of chronic stress exposure and examined as a potential effect measure modifier of the association between joint elevated NDT exposure and race/ethnicity. 


\section{Methods}

\subsection{The National Health and Nutrition Examination Surveys (NHANES) Data Set}

All biomarker (chemical and nonchemical stressor exposures), sociodemographic, lifestyle, and nutrient status data used in this analysis were extracted from NHANES. NHANES is a continuous survey that includes a physical examination (i.e., medical, dental, and physiological measurements), laboratory tests and an interview component collecting demographic, socioeconomic, dietary, and health-related data representative of the Unites States (U.S.) population. A stratified, multistage probability sample of the civilian, non-institutionalized U.S. population is used to select participants [18]. The 2003-2004 NHANES included 10,102 individuals including 5,152 women. For our analysis of female NHANES participants, the following exclusion criteria were applied: (1) not of reproductive age ([n=3,331 [reproductive age is defined here as 15 to 44 years]), (2) did not complete both the questionnaire and physical examinations $(n=64)$; $(3)$ were pregnant $(n=347)$, (4) self-identified their race/ethnicity as "other Hispanic" or "other race" ( $n=104)$, or (5) were missing biomarkers for blood $\mathrm{Pb}$ or blood $\mathrm{MeHg}(n=56)$. The final data set included 1,250 participants.

\subsection{Chemical NDT Stressor Exposure}

Whole blood $\mathrm{Hg}$ (total and inorganic) and $\mathrm{Pb}$ concentrations were determined using inductively coupled plasma-mass spectrometry [19]. Because inorganic $\mathrm{Hg}$ was below the detection limit $(0.3 \mu \mathrm{g} / \mathrm{L})$ in $75 \%$ of participants and total and inorganic $\mathrm{Hg}$ had different detection limits, we assumed that total $\mathrm{Hg}$ was entirely $\mathrm{MeHg}$. The detection limits were $0.2 \mu \mathrm{g} / \mathrm{dL}$ for $\mathrm{Pb}$ and $0.14 \mu \mathrm{g} / \mathrm{L}$ for total $\mathrm{Hg}$.

To evaluate joint $\mathrm{Pb}$ and $\mathrm{MeHg}$ exposure, we assumed dose-addition and calculated a hazard index (HI) for the combined $\mathrm{Pb}$ and $\mathrm{MeHg}$ dose by summing individual hazard quotients (HQs) [20]. Dose-addition assumes that both $\mathrm{Pb}$ and $\mathrm{MeHg}$ affect the same target, here the nervous system. HQs were calculated by comparing the blood $\mathrm{Pb}$ and blood $\mathrm{MeHg}$ biomarker concentrations with an NDT-specific health reference value (HRV). The following HRVs were used: $H R V_{\mathrm{Pb}}=1.76 \mu \mathrm{g} / \mathrm{dL}$, and $\mathrm{HRV}_{\mathrm{MeHg}}=5.8 \mu \mathrm{g} / \mathrm{L}$. The $\mathrm{HRV}_{\mathrm{MeHg}}$ was based on the maternal blood concentration associated with the U.S. Environmental Protection Agency's (EPA's) MeHg reference dose (RfD) $[21,22]$. The $\mathrm{HRV}_{\mathrm{Pb}}$ was based on a prospective cohort study reporting no intelligence quotient decrements at 3 years of age in Polish males with cord blood $\mathrm{Pb}$ values $\leq 1.21 \mu \mathrm{g} / \mathrm{dL}$, compared with males having cord blood $\mathrm{Pb}$ values $>1.21 \mu \mathrm{g} / \mathrm{dL}$ [23]. Using an average maternal-to-cord blood $\mathrm{Pb}$ ratio of 1.45 [24], we calculated the maternal blood $\mathrm{HRV}_{\mathrm{Pb}}(1.76 \mu \mathrm{g} / \mathrm{dL})$ equivalent to the cord blood $\mathrm{HRV}_{\mathrm{Pb}}(1.21 \mu \mathrm{g} / \mathrm{dL})$.

For the $i^{\text {th }}$ participant and the $j^{\text {th }}$ NDT, the NDT concentration was compared with the NDT-specific HRV to generate an HQ; these were summed across the two NDTs ( $\mathrm{Pb}$ and $\mathrm{MeHg}$ ) to evaluate joint NDT exposure for the $i^{\text {th }}$ participant (Equation 1):

$$
H I_{N D T_{i}}=\sum_{j=1}^{2} H Q_{i j}=\sum_{j=1}^{2} E_{i j} / H R V_{j}
$$


where $H I_{N D T i}$ is the hazard index from both $\mathrm{Pb}$ and $\mathrm{MeHg}$ for the $i^{\text {th }}$ participant; $H Q_{i j}$ is the hazard quotient for the $j^{\text {th }}$ NDT and the $i^{\text {th }}$ participant; $E_{i j}$ is the blood biomarker concentration for the $j^{\text {th }}$ NDT and the $i^{\text {th }}$ participant; and $H R V_{j}$ is the blood health reference value for the $j^{\text {th }}$ NDT.

All hazard values (i.e., $\mathrm{HQ}_{\mathrm{Pb}}, \mathrm{HQ}_{\mathrm{MeHg}}, \mathrm{HI}_{\mathrm{NDT}}$ ) were dichotomized with values $>1$ used as an indicator of elevated exposure (e.g., an $\mathrm{HI}_{\mathrm{NDT}}>1$ indicates elevated joint exposure to $\mathrm{Pb}$ and $\mathrm{MeHg}$ ).

Unlike the RfD-based $\mathrm{HRV}_{\mathrm{MeHg}}$, our literature-derived $\mathrm{HRV}_{\mathrm{Pb}}$ does not contain an uncertainty factor to account for intra-species variability. Therefore, we conducted a sensitivity analysis using lower and higher $\mathrm{HRV}_{\mathrm{Pb}}$ values based on California EPA's blood Pb benchmark in children of $1.0 \mu \mathrm{g} / \mathrm{dL}$ [25] and the Centers for Disease Control and Prevention's (CDC's) reference level of $5.0 \mu \mathrm{g} / \mathrm{dL}[26]$.

\subsection{AL Biomarkers}

The following ten AL biomarkers were chosen based on multi-system representation, availability, and previous research [27]: heart rate, mean systolic and diastolic blood pressure, homocysteine, body mass index (BMI), high-density lipoprotein (HDL) and total cholesterol, glycohemoglobin, C-reactive protein, and albumin (see online Supplementary Information for additional details). To evaluate potential chronic stress exposure, we computed an AL-clinical ${ }_{i}$ score for the $i^{\text {th }}$ participant by summing across 10 biomarker indicator variables $\left(\mathrm{BIV}_{\mathrm{ik}}\right)$ where the $k^{\text {th }}$ biomarker was assigned a "1" if its clinical criterion was exceeded or a " 0 " if not [28] (Equation 2, see online Supplementary Information, Tables S1 and S2):

$$
A L-\text { clinical }=\sum_{k=1}^{10} B I V_{i k}
$$

AL-clinical scores were used as an indicator of chronic stress exposure with scores of $0 ; 1$ or 2; and $\geq 3$, assumed to correspond to low, intermediate, and high chronic stress exposure, respectively. These cut-points were based on the distribution of AL-clinical scores and represent 28, 54, and 20\%, of women respectively. Participants missing AL biomarker data $(n=79)$ were excluded from analyses unless existing AL biomarker data led to an AL-clinical score of $\geq 3(n=11)$.

Other methods for calculating AL scores, including using empirical cut-points for classifying high risk and summation of $z$-scores, reportedly yield similar results [27,29,30]. We conducted a sensitivity analysis, calculating AL scores using empirically-determined high risk cut-points (AL-empirical) based on the distribution of each AL biomarker with high risk corresponding to the third quartile-high risk for HDL cholesterol and albumin correspond to the first quartile as lower values of these biomarkers indicate increased risk (see online Supplementary Information, Tables S1-S3).

\subsection{Data Analysis}

Statistical summaries were generated for all individual biomarkers and summary indices (i.e., AL, $\mathrm{HQ}_{\mathrm{Pb}}, \mathrm{HQ}_{\mathrm{MeHg}}, \mathrm{HI}_{\mathrm{NDT}}$ ) (see Tables $\mathrm{S} 1$ and $\mathrm{S} 2$ ). Logistic regression was used to evaluate the association between elevated joint NDT exposure $\left(\mathrm{HI}_{\mathrm{NDT}} \geq 1\right)$ and race/ethnicity. Confounding was evaluated using univariate and bivariate analyses (data not shown). Confounders were included in the multivariate model if they changed the association between elevated joint NDT exposure and 
race/ethnicity by $\geq 10 \%$ (see online Supplementary Information for a complete list of confounders and associated details). Chronic stress exposure was examined as an effect measure modifier via an interaction term in the final model (race/ethnicity $\times \mathrm{AL}$ ) and stratifying the multivariate model by AL-clinical groups. NHANES two-year sampling weights were used to produce unbiased, national estimates. Sampling weights do not account for differential birth rates, which could impact analyses. To evaluate the potential impact of differential birth rates on the associated between elevated joint NDT exposure and race/ethnicity, we performed an additional sensitivity analysis accounting for race/ethnicity- and age-specific birth rates based on methods from Axelrad and Cohen [31]; NHANES two-year sampling weights were multiplied by race/ethnicity- and age-specific birth rates and used in place of the original NHANES two-year sampling weights that were used in the main analysis.

All statistical analyses were conducted using SAS ${ }^{\circledR}$ software v9.3 (SAS Institute, Cary. NC, USA). Following NHANES analytic guidelines [18], we used procedures that accurately incorporated the stratification and multistage sampling: Proc SurveyMeans ${ }^{\circledR}$, Proc SurveyLogistic ${ }^{\circledR}$, and Proc SurveyFreq ${ }^{\circledR}$ survey procedures. Statistical significance was set at an alpha level of 0.05 and examined by the use of $p$-values and $95 \%$ Wald confidence intervals (95\% CIs).

\section{Results}

\subsection{Pb and MeHg Concentrations and Joint NDT Exposure}

Study participants were more likely to be Caucasian (weighted percentage $=74 \%$ ), born in the U.S. $(90 \%)$, greater than 28 years of age $(58 \%)$, college educated $(58 \%)$, nonsmokers $(72 \%)$, and have a normal iron status (74\%) (see Table 1). Overall median ( \pm interquartile range) $\mathrm{Pb}$ and $\mathrm{MeHg}$ blood concentrations were $0.9 \pm 0.6 \mu \mathrm{g} / \mathrm{dL}$ and $0.8 \pm 1.0 \mu \mathrm{g} / \mathrm{L}$, respectively. The median $\mathrm{HQ}_{\mathrm{Pb}}(0.5 \pm 0.3)$ was greater than the median $\mathrm{HQ}_{\mathrm{MeHg}}(0.1 \pm 0.1)$. Caucasians had a lower median $\mathrm{HQ}_{\mathrm{Pb}}$ compared to both African Americans and Mexican Americans $(p<0.05)$, but no race/ethnicity differences were detected for $\mathrm{HQ}_{\mathrm{MeHg}}$. The median $\mathrm{HI}_{\mathrm{NDT}}$ was $0.7 \pm 0.5$. As with $\mathrm{HQ}_{\mathrm{Pb}}$, Caucasians had a lower median $\mathrm{HI}_{\mathrm{NDT}}$ compared to both African Americans and Mexican Americans $(p<0.01$; see online Supplementary Information, Tables S1 and S2).

\subsection{Elevated Joint NDT Exposure and Chronic Stress}

Overall, $11 \%$ of participants had elevated blood $\mathrm{Pb}$ levels $\left(\mathrm{HQ}_{\mathrm{Pb}}>1\right)$, with Mexican Americans $(22 \%)$ having a higher proportion compared to Caucasians $(9 \%)(p<0.05$; see Table 1$)$. Only $2 \%$ of all participants had elevated blood MeHg levels; no differences were detected by race/ethnicity. One-quarter of all study participants had elevated joint NDT exposure levels and $20 \%$ were classified as having a high AL, with African Americans having higher proportions compared to Caucasians for both elevated NDT exposure and high $\operatorname{AL}(p<0.05)$.

Adjusting for country of birth, age, education, smoking status, and iron status had a mixed effect on the association between elevated joint NDT exposure and race/ethnicity. The odds ratio (OR) increased nearly $30 \%$ for African Americans after accounting for relevant confounders (unadjusted OR $[95 \% \mathrm{CI}]=1.7[1.0,2.6]$; adjusted OR $[95 \% \mathrm{CI}]=2.2[1.4,3.3])$ but decreased by $43 \%$ for Mexican Americans (unadjusted OR $[95 \% \mathrm{CI}]=2.0[1.3,3.0]$; adjusted OR $[95 \% \mathrm{CI}]=1.4[0.7,2.6]$ ) 
(see Table 2). In addition to differences detected by race/ethnicity in the multivariate model, participants were more likely to have elevated joint NDT exposure if they were (1) foreign born (adjusted OR [95\% CI] = $3.3[1.6,6.8]$ ); (2) older, including 20-28 years of age $(1.8[1.0,3.2]$ ) and $29-44$ years of age $(3.5[2.4,5.1])$; or (3) smokers $(3.0[1.7,5.0])$. An inverse association was detected between elevated joint NDT exposures and iron status, with participants having an abnormal iron status less likely to have elevated joint NDT exposure (adjusted OR $[95 \% \mathrm{CI}]=0.6[0.5,0.8]$ ).

\subsection{Effect Measure Modification by $A L$}

Effect measure modification of the association between elevated joint NDT exposure and race/ethnicity was detected by $\mathrm{AL}$. The overall interaction term race/ethnicity $\times \mathrm{AL}$ was not statistically significant $(p=0.1)$ but the interaction between African Americans and high AL was statistically significant $(p=0.02)$. Although no racial/ethnic differences were observed among participants with low ALs, the largest ORs were noted among participants with high ALs (adjusted OR $[95 \% \mathrm{CI}]=4.3[2.0,9.5]$ and $4.2[1.3,14.1]$ for African Americans and Mexican Americans, respectively) (see Table 2, Figure 1). Although an increasing trend across AL groups was not detected for age, ORs increased with increasing age for all AL groups with the largest ORs observed among participants with high ALs (adjusted ORs $[95 \% \mathrm{CI}]=19.1[1.8,201.6]$ and $33.3[3.6,305.5]$ for ages 20-28 years and 29-44 years, respectively). An inverse association between country of birth and elevated joint NDT exposure was observed across AL groups among foreign-born participants with the largest ORs observed among foreign-born participants with low ALs (adjusted OR [95\% CI] = $10.3[2.5,43.2]$ ).

\subsection{Sensitivity Analyses}

The main analysis assumed equal birth rates across all race/ethnicity and age categories; therefore, we performed a sensitivity analysis accounting for different birth rate trends across race/ethnicity and age. Using NHANES two-year sampling weights adjusted for race/ethnicity — and age-specific birth rates resulted in similar trends as noted in the main analysis but lower ORs for the association between elevated joint NDT exposure and race/ethnicity particularly among Mexican Americans with high AL-clinical scores (see online Supplementary Information, Table S4).

We also examined differences in ORs using AL-empirical scores and lower and higher $\mathrm{HRV}_{\mathrm{Pb}} \mathrm{S}$ $(1.0 \mu \mathrm{g} / \mathrm{dL}$ and $5.0 \mu \mathrm{g} / \mathrm{dL}$, respectively). We found comparable relationships for African Americans using AL-empirical scores (see online Supplementary Information, Table S3). Larger ORs were noted for the high $\mathrm{AL}$ group using a $\mathrm{HRV}_{\mathrm{Pb}}$ of $5.0 \mu \mathrm{g} / \mathrm{dL}$, but the results were less stable. Mexican Americans with a high AL tended to have larger ORs, compared to other AL groups, regardless of the AL approach (AL-clinical or AL-empirical) or HRV used $(1.0 \mu \mathrm{g} / \mathrm{dL}$ and $5.0 \mu \mathrm{g} / \mathrm{dL}$ ), except when AL-empirical scores and the higher $\mathrm{HRV}_{\mathrm{Pb}}$ were used in combination (see online Supplementary Information, Table S3). 
Table 1. Characteristics of nonpregnant, reproductive-aged (15-44 years) women from NHANES 2003-2004 by indicators of elevated neurodevelopmental toxicant (NDT) exposure ${ }^{\mathrm{a}}$ and allostatic load ${ }^{\mathrm{b}}$.

\begin{tabular}{|c|c|c|c|c|c|c|c|}
\hline & \multirow{2}{*}{$\begin{array}{c}\text { All Women } \\
n \\
\text { (Weighted \%) }\end{array}$} & \multicolumn{3}{|c|}{ Indicators of Elevated NDT Exposure $^{\text {a }}$} & \multicolumn{3}{|c|}{ Allostatic Load $^{\text {b }}$} \\
\hline & & $\begin{array}{c}H Q_{\mathrm{Pb}}>1 \\
n(\text { Weighted \%) }\end{array}$ & $\begin{array}{c}\mathrm{HQ}_{\mathrm{MeHg}}>1 \\
n(\text { Weighted \%) }\end{array}$ & $\begin{array}{c}\mathrm{HI}_{\mathrm{NDT}}>1 \\
n(\text { Weighted \%) }\end{array}$ & $\begin{array}{c}\text { Low } \\
n \text { (Weighted \%) }\end{array}$ & $\begin{array}{c}\text { Intermediate } \\
n \text { (Weighted \%) }\end{array}$ & $\begin{array}{c}\text { High } \\
n \text { (Weighted \%) }\end{array}$ \\
\hline Total Population & 1,250 & $159(11)$ & $19(2)$ & $324(25)$ & $330(28)$ & $632(54)$ & $210(20)$ \\
\hline \multicolumn{8}{|c|}{ Race/Ethnicity } \\
\hline Caucasian & $551(74)$ & $46(9)$ & $10(2)$ & $119(22)$ & $156(28) *$ & $274(54)$ & $88(19)$ \\
\hline African American & $373(15)$ & $52(14)$ & $6(2)$ & $104(32) *$ & $86(18)$ & $194(55)$ & $67(26) *$ \\
\hline Mexican American & $326(11)$ & $61(22) *$ & $3(1)$ & $101(36) *$ & $88(21)$ & $164(56)$ & $55(23)$ \\
\hline \multicolumn{8}{|c|}{ Country of Birth } \\
\hline United States & $1,049(90)$ & $92(8)$ & $15(2) *$ & $225(23)$ & $278(25)$ & $533(54)$ & $175(20)$ \\
\hline Foreign & $201(10)$ & $67(33) *$ & $4(2)$ & $99(48) *$ & $52(26)$ & $99(52)$ & $35(22)$ \\
\hline \multicolumn{8}{|c|}{ Age (years) } \\
\hline $15-19$ & $520(16)$ & $43(5)$ & $4(1)$ & $79(11)$ & $191(43)$ & $254(49)$ & $40(8)$ \\
\hline $20-28$ & $252(26)$ & $29(9)$ & $3(1)$ & $59(20) *$ & $66(30) *$ & $136(58) *$ & $31(12) *$ \\
\hline $29-44$ & $478(58)$ & $87(14) *$ & $12(2)$ & $184(33) *$ & $73(19) *$ & $242(54)$ & $139(28) *$ \\
\hline \multicolumn{8}{|c|}{ Highest Education $^{c}$} \\
\hline Less than high school graduate & $327(15)$ & $70(22) *$ & $4(1)$ & $114(39)$ & $68(18) *$ & $170(53)$ & $69(29) *$ \\
\hline High school graduate & $302(27)$ & $37(11)$ & $3(1)$ & $67(21)$ & $74(20) *$ & $152(53)$ & $55(27) *$ \\
\hline Some college & $383(35)$ & $30(7)$ & $3(1)$ & $75(19)$ & $107(28) *$ & $197(56)$ & $54(17)$ \\
\hline College graduate or above & $194(23)$ & $17(8)$ & $9(5) *$ & $57(31) *$ & $67(33)$ & $90(54)$ & $26(13)$ \\
\hline \multicolumn{8}{|c|}{ Smoking Status (serum cotinine) } \\
\hline Nonsmoker $(\leq 10 \mathrm{ng} / \mathrm{mL})$ & $943(72)$ & $100(8)$ & $14(2)$ & $214(22)$ & $270(27)$ & $485(54)$ & 147 (19) \\
\hline Smoker $(>10 \mathrm{ng} / \mathrm{mL})$ & $282(28)$ & $56(18) *$ & $5(2)$ & $105(37) *$ & $60(21) *$ & $147(55)$ & $62(25) *$ \\
\hline
\end{tabular}


Table 1. Cont.

\begin{tabular}{|c|c|c|c|c|c|c|c|}
\hline & \multirow{2}{*}{$\begin{array}{c}\text { All Women } \\
n \\
\text { (Weighted \%) }\end{array}$} & \multicolumn{3}{|c|}{ Indicators of Elevated NDT Exposure ${ }^{\text {a }}$} & \multicolumn{3}{|c|}{ Allostatic Load $^{\text {b }}$} \\
\hline & & $\begin{array}{c}H Q_{P b}>1 \\
n(\text { Weighted \%) }\end{array}$ & $\begin{array}{c}\mathrm{HQ}_{\mathrm{MeHg}}>1 \\
n(\text { Weighted \%) }\end{array}$ & $\begin{array}{c}\mathrm{HI}_{\mathrm{NDT}}>1 \\
\boldsymbol{n}(\text { Weighted \%) }\end{array}$ & $\begin{array}{c}\text { Low } \\
n \text { (Weighted \%) }\end{array}$ & $\begin{array}{c}\text { Intermediate } \\
n \text { (Weighted \%) }\end{array}$ & $\begin{array}{c}\text { High } \\
n(\text { Weighted \%) }\end{array}$ \\
\hline Total Population & 1,250 & $159(11)$ & $19(2)$ & $324(25)$ & $330(28)$ & $632(54)$ & $210(20)$ \\
\hline \multicolumn{8}{|c|}{ Iron Status Indicator $^{\mathrm{d}}$} \\
\hline Normal & $870(74)$ & $105(11)$ & $17(2)$ & $235(28)$ & $247(27) *$ & $449(55)$ & $122(18) *$ \\
\hline Abnormal & $379(26)$ & $54(11)$ & $2(0)$ & $89(21)$ & $83(19)$ & $183(52)$ & $87(28)$ \\
\hline
\end{tabular}

Notes: ${ }^{a}$ Hazard quotients (HQs) were calculated by dividing blood concentrations of lead (Pb) and methyl mercury (MeHg) by $1.76 \mu \mathrm{g} / \mathrm{dL}$ and $5.8 \mu \mathrm{g} / \mathrm{L}$, respectively. The hazard index

(HI) was calculated by summing individual hazard quotients for $\mathrm{Pb}$ and $\mathrm{MeHg}$ (Equation 1). ${ }^{\mathrm{b}}$ Allostatic load, a measure of physiological dysregulation, was used as an indicator of chronic stress exposure and was estimated based on the categorical classification of AL-clinical scores $(0=$ Low; $1-2=$ Intermediate; $\geq 3=$ High $)$ and calculated by summing the number of 10 biomarkers above clinical high risk criteria (Equation 2). ${ }^{\mathrm{c}}$ If the participant was $<18$ years of age, head of household status was used. ${ }^{\mathrm{d}}$ Iron status was determined abnormal if any two of the following conditions were met: (1) serum ferritin $<15 \mathrm{ng} / \mathrm{mL}$, (2) transferrin saturation $<16 \%$, (3) red blood cell distribution width $>15 \%$, or (4) erythrocyte protoporphyrin $>50 \mu \mathrm{g} / \mathrm{dL}$ red blood cells. * Denotes statistically significant within group differences between the indicated percentage and the lowest (or highest) percentage $(p<0.05)$ (e.g., Mexican Americans had a statistically significant higher percentage of women with an $\mathrm{HQ}_{\mathrm{Pb}}>1$ relative to Caucasians).

Table 2. Odds Ratios (ORs) and 95\% Wald Confidence Intervals (CIs) for the association between elevated joint neurodevelopmental toxicant exposure $^{\mathrm{a}}$ and race/ethnicity among nonpregnant, reproductive-aged (15-44 years) women from NHANES 2003-2004.

\begin{tabular}{|c|c|c|c|c|c|}
\hline & \multicolumn{5}{|c|}{ OR $(95 \% \mathrm{CI})$} \\
\hline & \multirow[b]{2}{*}{ Univariate } & \multirow[b]{2}{*}{ Multivariate } & \multicolumn{3}{|c|}{ Multivariate by Allostatic Load ${ }^{\text {b }}$} \\
\hline & & & Low & Intermediate & High \\
\hline & & $n=1,181$ & $n=316$ & $n=609$ & $n=203$ \\
\hline Race/Ethnicity & & & $n=1,250$ & & \\
\hline Caucasian & 1.0 & 1.0 & 1.0 & 1.0 & 1.0 \\
\hline African American & $1.7(1.0,2.6)$ & $2.2(1.4,3.3)$ & $1.2(0.5,2.7)$ & $2.7(1.6,4.5)$ & $4.3(2.0,9.5)$ \\
\hline Mexican American & $2.0(1.3,3.0)$ & $1.4(0.7,2.6)$ & $0.8(0.2,4.1)$ & $1.9(0.9,4.0)$ & $4.2(1.3,14.1)$ \\
\hline
\end{tabular}


Table 2. Cont.

\begin{tabular}{|c|c|c|c|c|c|}
\hline & \multicolumn{5}{|c|}{ OR $(95 \%$ CI) } \\
\hline & \multirow[b]{2}{*}{ Univariate } & \multirow[b]{2}{*}{ Multivariate } & \multicolumn{3}{|c|}{ Multivariate by Allostatic Load ${ }^{\text {b }}$} \\
\hline & & & Low & Intermediate & High \\
\hline & & $n=1,181$ & $n=316$ & $n=609$ & $n=203$ \\
\hline Country of Birth & & & $n=1,250$ & & \\
\hline United States & 1.0 & 1.0 & 1.0 & 1.0 & 1.0 \\
\hline Foreign & $3.1(1.8,5.4)$ & $3.3(1.6,6.8)$ & $10.3(2.5,43.2)$ & $2.2(1.2,4.3)$ & $1.8(0.4,8.1)$ \\
\hline Age (years) & & & $n=1,250$ & & \\
\hline $15-19$ & 1.0 & 1.0 & 1.0 & 1.0 & 1.0 \\
\hline $20-28$ & $2.1(1.4,3.2)$ & $1.8(1.0,3.2)$ & $2.5(0.9,7.4)$ & $1.6(0.6,4.2)$ & $19.1(1.8,201.6)$ \\
\hline $29-44$ & $4.1(3.1,5.5)$ & $3.5(2.4,5.1)$ & $6.3(2.2,17.7)$ & $3.9(1.8,8.4)$ & $33.3(3.6,305.5)$ \\
\hline Highest Education $^{\mathrm{c}}$ & & & $n=1,250$ & & \\
\hline Less than high school graduate & $1.4(0.8,2.5)$ & $0.8(0.5,1.5)$ & $1.9(0.5,7.6)$ & $0.7(0.3,1.7)$ & $0.6(0.1,2.9)$ \\
\hline High school graduate & $0.6(0.3,1.0)$ & $0.4(0.2,0.6)$ & $0.6(0.2,1.8)$ & $0.4(0.2,0.6)$ & $0.5(0.1,4.3)$ \\
\hline Some college & $0.5(0.3,0.8)$ & $0.8(0.5,1.5)$ & $0.8(0.4,1.7)$ & $0.2(0.1,0.4)$ & $0.7(0.2,2.8)$ \\
\hline College graduate or above & 1.0 & 1.0 & 1.0 & 1.0 & 1.0 \\
\hline Smoking Status (serum cotinine) & & & $n=1,225$ & & \\
\hline Nonsmoker ( $\leq 10 \mathrm{ng} / \mathrm{mL})$ & 1.0 & 1.0 & 1.0 & 1.0 & 1.0 \\
\hline Smoker (>10 ng/mL) & $2.0(1.4,3.0)$ & $3.0(1.7,5.0)$ & $2.0(0.8,4.6)$ & $3.8(2.0,7.4)$ & $3.4(1.1,10.6)$ \\
\hline Iron Status Indicator $^{d}$ & & & $n=1,250$ & & \\
\hline Normal & 1.0 & 1.0 & 1.0 & 1.0 & 1.0 \\
\hline Abnormal & $0.7(0.5,1.0)$ & $0.6(0.5,0.8)$ & $1.1(0.5,2.4)$ & $0.5(0.3,0.7)$ & $0.7(0.3,1.4)$ \\
\hline
\end{tabular}

Notes: ${ }^{a}$ Elevated NDT exposure was defined as having a hazard index (HI) for joint lead $(\mathrm{Pb})$ and methyl mercury $(\mathrm{MeHg})$ greater than one $\left(\mathrm{HI} \mathrm{NDT}_{\mathrm{N}}>1\right)$. The $\mathrm{HI}$ was calculated by summing individual hazard quotients (HQs) for $\mathrm{Pb}$ and $\mathrm{MeHg}$ (Equation 1 ). HQs were calculated by dividing blood concentrations of $\mathrm{Pb}$ and $\mathrm{MeHg}$ by $1.76 \mu \mathrm{g} / \mathrm{dL}$ and $5.8 \mu \mathrm{g} / \mathrm{L}$, respectively. ${ }^{\mathrm{b}}$ Allostatic load (AL), a measure of physiological dysregulation, was used as an indicator of chronic stress exposure and was estimated based on the categorical classification of AL-clinical scores ( $0=$ Low; $1-2=$ Intermediate; $>2=$ High) and calculated by summing the number of 10 biomarkers above clinical high risk criteria (Equation 2$)$. ${ }^{\mathrm{c}}$ If the participant was $<18$ years of age, status of the head of household was used. ${ }^{\mathrm{d}}$ Iron status was determined to be abnormal if any two of the following conditions were met: (1) serum ferritin $<15 \mathrm{ng} / \mathrm{mL},(2)$ transferrin saturation $<16 \%$, (3) red blood cell distribution width $>15 \%$, or (4) erythrocyte protoporphyrin $>50 \mu \mathrm{g} / \mathrm{dL}$ red blood cells. 


\section{Discussion}

Our analysis suggests that $25 \%$ of nonpregnant, reproductive-aged women in the 2003-2004 NHANES had blood levels of combined $\mathrm{Pb}$ and $\mathrm{MeHg}$ that may be of concern for adverse neurodevelopment in offspring (see Table 1). We identified race/ethnicity as one of the strongest individual-level predictors of elevated joint NDT exposure to $\mathrm{Pb}$ and $\mathrm{MeHg}$. While numerous studies have estimated exposure distributions of $\mathrm{Pb}$ and $\mathrm{MeHg}$ independently [32,33], consideration of the joint exposure distribution of these NDTs is rare [5,6]. Our study also found that AL, an indicator of chronic stress, modified the association between race/ethnicity and elevated joint NDT exposure (see Table 2; Figure 1). We found no differences across racial/ethnic groups for the likelihood of having elevated joint NDT exposure among participants with low chronic stress. Whether chronic stress increases vulnerability to $\mathrm{Pb}$ - and/or $\mathrm{MeHg}$-mediated neurodevelopmental toxicity is unclear. Our analysis, however, provides evidence of joint exposure to chronic stress and multiple NDTs and suggests that racial/ethnic disparities exist for both chemical and nonchemical exposures independent of other risk factors including age and education.

The HI is a screening approach that examines the joint hazard associated with multiple chemicals under an assumption of dose-addition and is useful for the preliminary prioritization of stressors [20]. There is limited information available regarding the joint neurotoxicity of $\mathrm{Pb}$ and $\mathrm{MeHg}$; therefore, there is uncertainty associated with the assumption of dose-addition but additivity is consistent with CDC recommendations for this combination of NDTs [34]. Although the current analysis only examined a chemical mixture of two metals with a similar endpoint, this approach could be extended to include additional chemical stressors. It is currently unclear whether nonchemical stressors, such as measures of physiological dysregulation, can be directly incorporated into the HI. In general, effect measure modification can be used to examine vulnerable populations and identify potential interactions. We examined effect measure modification by potential chronic stress exposure on the main effect using stratification and tests for interactions.

The HRVs used in the HI calculation for the current analysis are similar to Biomonitoring Equivalents in that biomarker concentrations are compared with risk assessment-based values to facilitate their interpretation in a public health risk context [35]. The $\mathrm{HRV}_{\mathrm{MeHg}}$ was based on the U.S. EPA RfD. RfDs are "estimates of daily exposure for the human population... likely to be without an appreciable risk of deleterious effects over a lifetime"; such values have been thoroughly peer reviewed [36]. Because no scientific consensus exists on a "safe value" of blood $\mathrm{Pb}$, we chose a value identified in the literature that is unlikely to be associated with adverse neurodevelopment. The $\mathrm{HRV}_{\mathrm{Pb}}$ used in the current analysis has not been as thoroughly vetted as the $\mathrm{HRV}_{\mathrm{MeHg}}$ and does not include an uncertainty factor accounting for intra-person variability. We conducted sensitivity analyses using higher and lower $H R V_{\mathrm{Pb}}$ to highlight some of the uncertainty associated with our $\mathrm{HRV}_{\mathrm{Pb}}$ and found similar results across all $\mathrm{HRV}_{\mathrm{Pb}} \mathrm{S}$.

The $\mathrm{HI}$ is health conservative, identifying populations estimated to have an HQ $>1$ for any single chemical, as well as $<1$ for all chemicals individually, yet have an HI $>1$ when HQs are combined across stressors. For example, half of the $25 \%$ of women identified as having elevated joint NDT exposure, based on an $\mathrm{HI}_{\mathrm{NDT}}>1$, had $\mathrm{HQs}<1$ for $\mathrm{Pb}$ or $\mathrm{MeHg}$, and less than $1 \%$ of these women $(n=3)$ had HQs $>1$ for both $\mathrm{Pb}$ and $\mathrm{MeHg}$ independently. Additionally, the HI approach accounts for 
the relative toxicities of stressors, potentially allowing for prioritization of stressors and identification of vulnerable populations. This is exemplified in our analysis: $\mathrm{Pb}$ accounted for more than $70 \%$ of the total $\mathrm{HI}_{\mathrm{NDT}}$ and African Americans were more likely than Caucasians to experience elevated joint NDT exposure. Thompson and Boekelheide [5] reported that, although statistically significant differences by race/ethnicity were not observed for the odds of greater than median exposure to $\mathrm{Pb}$, $\mathrm{MeHg}$, or PCBs, non-Caucasians had larger adjusted ORs compared to Caucasians, and Hispanics had smaller adjusted ORs compared to other non-Caucasians. Our results underscore the importance of monitoring chemical and nonchemical stressor exposures.

Figure 1. Adjusted a odds ratios (ORs) and 95\% Wald confidence intervals (CIs) for the association between elevated joint neurodevelopmental toxicant (NDT) exposure ${ }^{b}$ and race/ethnicity stratified by allostatic load ${ }^{\mathrm{c}}$ among nonpregnant, reproductive-aged (15-44 years) women from NHANES 2003-2004.

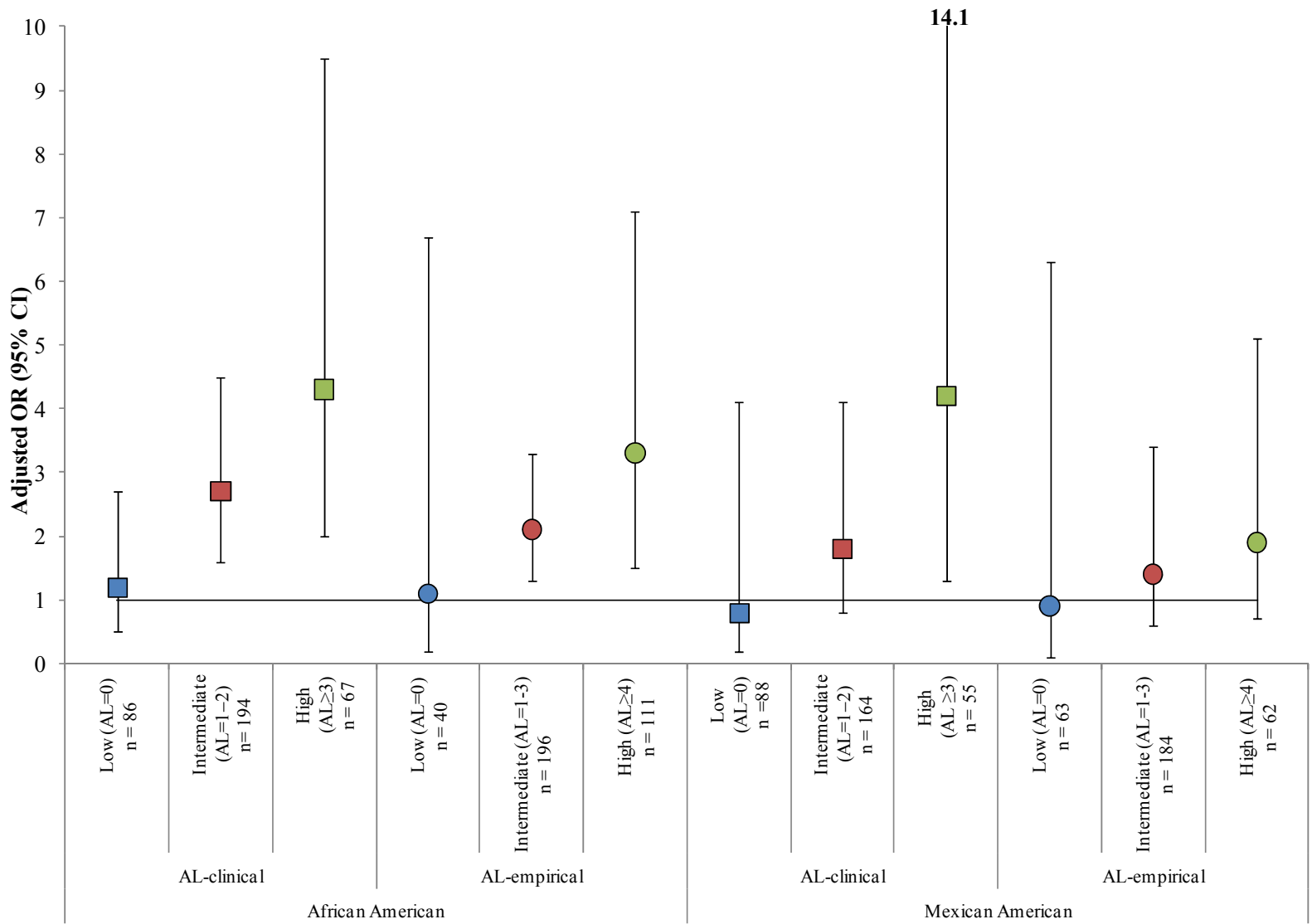

Notes: ${ }^{a}$ Adjusted for age, country of birth, head of household highest education, smoking status, and iron status. ${ }^{\mathrm{b}}$ Elevated NDT exposure was defined as having a hazard index $(\mathrm{HI})$ for joint lead $(\mathrm{Pb})$ and methyl mercury $(\mathrm{MeHg})$ greater than one $\left(\mathrm{HI}_{\mathrm{NDT}}>1\right)$. The $\mathrm{HI}$ was calculated by summing individual hazard quotients (HQs) for $\mathrm{Pb}$ and $\mathrm{MeHg}$ (Equation 1). HQs were calculated by dividing blood concentrations of $\mathrm{Pb}$ and $\mathrm{MeHg}$ by $1.76 \mu \mathrm{g} / \mathrm{dL}$ and $5.8 \mu \mathrm{g} / \mathrm{L}$, respectively. ${ }^{\mathrm{c}}$ Allostatic load (AL), a measure of physiological dysregulation, was used as an indicator of chronic stress exposure and was estimated based on the categorical classification of AL scores and calculated by summing the number of 10 biomarkers above clinical (AL-clinical) or empirical (AL-empirical) high risk criteria (Equation 2). Squares indicate adjusted ORs for AL-clinical. Circles indicate adjusted ORs for AL-empirical. Regardless of AL method: blue indicates low AL (low chronic stress exposure), red indicates intermediate AL (intermediate chronic stress exposure), and green indicates high AL (high chronic stress exposure). 
Exposure disparities across and within racial/ethnic groups may be associated with differential vulnerability. Vulnerability can imply that an individual or population differs from the "general" population because of increased exposure, a different dose-response curve, or a differential ability to resist or recover from stressor exposures [37]. For example, we found that, although $36 \%$ of all Mexican American women had elevated joint NDT exposure (see Table 1), there were statistically significant differences among Mexican Americans by country of birth (U.S. born $=23 \%$ and foreign-born $=51 \%$; unadjusted OR $[95 \% \mathrm{CI}]=3.6[2.2,6.1]$, data not shown). Nelson, et al. [38] also reported differences in environmental chemical concentrations between foreign-born and U.S. born Mexican Americans. Although Crimmins, et al. [39] found that U.S. born Mexican Americans had higher ALs compared to both Caucasians and foreign-born Mexican Americans, we did not detect statistically significant differences among Mexican Americans by country of birth for the likelihood of having high chronic stress. Although country of birth was a statistically significant variable in our multivariate model and a decrease in adjusted ORs was noted with increasing AL score (see Table 2), we did not have a large enough sample size to further investigate the potential interaction between race/ethnicity and country of birth and any modifying effect by potential chronic stress exposure. A strength of this study was the large number of variables available for examination as possible confounders of the main effect (see online Supplementary Information for a complete list); therefore, the potential for residual confounding was decreased but perhaps not entirely eliminated as some variables of interest may be mismeasured and data on other possible confounders were not available (e.g., occupation, genetic susceptibility, etc.). Results from our analysis and previous studies [5,38,39] reinforce the need to examine chemical and nonchemical stressor exposures across and within sociodemographics in future epidemiological studies and cumulative risk assessments (CRAs) as these factors may contribute to differential vulnerability.

Sociodemographic risk factors may also influence birth rates [40]. Using sampling weights adjusted for race/ethnicity- and age-specific birth rates, we observed comparable results to the main analysis, but most ORs were attenuated (see online Supplementary Information, Table S4). The smaller adjusted ORs were likely due to the combination of different birth rates, NDT exposures and AL scores across racial/ethnic groups. For example, although the percentage of women with elevated joint NDT exposure and high chronic stress increased with age across all racial/ethnic groups, birth rates were higher among younger non-Caucasian and older Caucasian women. While the use of sampling weights adjusted for race/ethnicity- and age-specific birth rates minimally affected the logistic regression results, the estimated number of women with elevated joint NDT exposure was lower (only about 8\% of the estimate based on unadjusted birth rates, data not shown). This sensitivity analysis supports the main results that African American women with high chronic stress had higher odds of elevated joint NDT exposure. This analysis also highlights the importance of accounting for differential outcome rates (e.g., birth rates) when estimating associations and/or the number of individuals potentially at increased risk.

Exposure measurement is a primary challenge in epidemiological studies and CRAs. Cross-sectional data, such as NHANES, have limitations that can limit their utility in broader applications. For example, the potential for reverse causation due to uncertain temporality (i.e., ensuring that a putative causal agent preceded the exposure) is often a concern. This was not an issue in our analysis as we were assessing whether race/ethnicity, which is an immutable factor that is 
constant over time, was a predictor of elevated joint NDT exposure. There is, however, some uncertainty due to the assumption that NDT exposures detected from one-time measures of $\mathrm{Pb}$ and $\mathrm{MeHg}$ in non-pregnant reproductive age women are representative of potential future maternal and in utero exposures. Both $\mathrm{Pb}$ and $\mathrm{MeHg}$ have relatively short half-lives in blood (approximately 1 and 2 months, respectively) [13,14]; therefore, concentrations in the current analysis reflect recent exposures. Additionally, chemical stressor exposures likely differ during pregnancy. For example, blood $\mathrm{Pb}$ may increase during pregnancy due to increased bone turnover [41]. In contrast, levels of many environmental chemicals are reportedly lower among pregnant women [42] potentially due to physiological changes (e.g., increased blood volume) and lifestyle and behavioral modifications. The use of one-time biomarker samples can result in measurement error, which may limit our ability to fully elucidate independent associations and effect measure modification that may be evident in this population.

In addition to the limitations associated with one-time measurements, AL scores, used to indicate chronic stress exposure, were limited by the lack of availability of the primary stress mediators (i.e., cortisol, catecholamines), although these biomarkers also have limitations [43]. Measures of physiological dysregulation, such as AL, are not ideal indicators of chronic stress exposure because non-chronic stress factors such as genetics and lifestyle choices could also result in physiological dysregulation and, therefore, a higher AL [16]. Regardless of the cause(s) associated with an increased AL, physiological dysregulation may be associated with increased vulnerability to adverse effects associated with exposure to chemical stressors [44]. We excluded pregnant women from our analysis because of uncertainty whether AL could serve as a surrogate of in utero chronic stress exposure due to the physiological changes that occur throughout pregnancy $[45,46]$. However, it is reasonable to assume that nonpregnant women of reproductive age identified as having elevated levels of neurodevelopmental stressors could also have elevated levels during pregnancy, and this may be an indication of potential concern for their offspring.

Psychosocial stressors are difficult to measure, as evidenced by various reported approaches for assessing chronic stress; such as exposure to violence [47], the University of California, Los Angeles Life Stress Interview [48], the Perceived Stress Scale [49], depression [50], and AL [27]. No NHANES interview questions among reproductive-aged women pertain to chronic stress; therefore, we used AL as an indicator of chronic stress exposure. Although quantitative measures of physiological dysregulation, such as AL, may decrease reporting error and increase reproducibility and comparability across studies, having subjective measures of chronic stress, such as those listed above, would be useful for cross-validation. There are, however, no standard clinical high risk criteria, and empirical high risk cut-points vary depending on the underlying population characteristics, making it difficult to compare results across studies and to make generalizations across populations. In our analysis, empirical cut-points were influenced by Caucasians, the predominant population in NHANES. Caucasians had lower values for nearly all AL biomarkers compared to African Americans but similar values to Mexican Americans (see online Supplementary Information, Table S2). Therefore, we believe that AL-clinical, as used in our main analysis, was better suited for evaluating racial/ethnic differences.

Additional limitations have been associated with the operationalization of AL [27,51]. Traditional $\mathrm{AL}$ approaches dichotomize AL biomarkers, but there is likely a continuum of risk that may not lend 
itself well to dichotomous classification. An associated limitation is not accounting for the bi-directionality of physiological dysregulation (i.e., assuming that potential concern exists only above a defined cut-point). Levels outside the normal physiological range (higher or lower) may be associated with dysregulation and chronic stress (e.g., low BMI) [52]. Future research should examine whether AL is a useful measure of chronic stress across the lifespan, especially in reproductive-aged women before, during, and after pregnancy, as well as account for the bi-directionality and continuum of harm associated with individual AL biomarkers.

\section{Conclusions}

Chemical and nonchemical stressors are rarely examined jointly in human health studies or risk assessments. Our results suggest that chronic stress modified the association between race/ethnicity and elevated joint NDT exposure. These results support the use of existing methods (e.g., HI and AL) and data sources (e.g., NHANES) to examine cumulative chemical and nonchemical stressor exposures, potentially elucidating causal associations and explaining equivocal results reported across epidemiological studies for some environmental chemicals. Including chronic stress in the evaluation of joint NDT exposure allowed for the identification of potentially vulnerable populations, which may require further evaluation.

\section{Acknowledgments}

We would like to thank Rita Schoeny and Deborah Cory-Slechta for their helpful comments on earlier versions of this manuscript. This project was supported in part by an appointment to the Research Participation Program at the National Center for Environmental Assessment, Office of Research and Development, U.S. EPA, administered by ORISE through an interagency agreement between the U.S. Department of Energy and U.S. EPA. The views expressed in this article are those of the authors and do not necessarily reflect the views or policies of the EPA.

\section{Author Contributions}

Amanda M. Evans, Glenn E. Rice, Linda K. Teuschler, and J. Michael Wright conceived and designed the study. Amanda M. Evans wrote the first draft of the manuscript and Amanda M. Evans, Glenn E. Rice, Linda K. Teuschler, and J. Michael Wright revised the manuscript.

\section{Conflicts of Interest}

The authors declare no conflict of interest.

\section{References}

1. Lin, C.C.; Chen, Y.C.; Su, F.C.; Lin, C.M.; Liao, H.F.; Hwang, Y.H.; Hsieh, W.S.; Jeng, S.F.; $\mathrm{Su}$, Y.N.; Chen, P.C. In utero exposure to environmental lead and manganese and neurodevelopment at 2 years of age. Environ. Res. 2013, 123, 52-57.

2. Jones, E.A.; Wright, J.M.; Rice, G.; Buckley, B.T.; Magsumbol, M.S.; Barr, D.B.; Williams, B.L. Metal exposures in an inner-city neonatal population. Environ. Int. 2010, 36, 649-654. 
3. Lanphear, B.P.; Hornung, R.; Khoury, J.; Yolton, K.; Baghurst, P.; Bellinger, D.C.; Canfield, R.L.; Dietrich, K.N.; Bornschein, R.; Greene, T. Low-level environmental lead exposure and children's intellectual function: An international pooled analysis. Environ. Health Persp. 2005, 113, 894-899.

4. Grandjean, P.; Weihe, P.; Nielsen, F.; Heinzow, B.; Debes, F.; Budtz-Jorgensen, E. Neurobehavioral deficits at age 7 years associated with prenatal exposure to toxicants from maternal seafood diet. Neurotoxicol. Teratol. 2012, 34, 466-472.

5. Thompson, M.R.; Boekelheide, K. Multiple environmental chemical exposures to lead, mercury and polychlorinated biphenyls among childbearing-aged women (NHANES 1999-2004): Body burden and risk factors. Environ. Res. 2013, 121, 23-30.

6. Yorifuji, T.; Debes, F.; Weihe, P.; Grandjean, P. Prenatal exposure to lead and cognitive deficit in 7- and 14-year-old children in the presence of concomitant exposure to similar molar concentration of methylmercury. Neurotoxicol. Teratol. 2011, 33, 205-211.

7. Bergman, K.; Sarkar, P.; Glover, V.; O’Connor, T.G. Maternal prenatal cortisol and infant cognitive development: Moderation by infant-mother attachment. Biol. Psychiatry 2010, 67, 1026-1032.

8. Jones, R.L.; Homa, D.M.; Meyer, P.A.; Brody, D.J.; Caldwell, K.L.; Pirkle, J.L.; Brown, M.J. Trends in blood lead levels and blood lead testing among US children aged 1 to 5 years, 1988-2004. Pediatrics 2009, 123, e376-e385.

9. Geronimus, A.T.; Hicken, M.; Keene, D.; Bound, J. "Weathering" and age patterns of allostatic load scores among blacks and whites in the United States. Am. J. Public Health 2006, 96, 826-833.

10. Peters, J.L.; Weisskopf, M.G.; Avron Spiro, J.S., III; Sparrow, D.; Nie, H.; Hu, H.; Wright, R.O.; Wright, R.J. Interaction of stress, lead burden, and age on cognition in older men: The VA Normative Aging Study. Environ. Health Persp. 2010, 118, 505-510.

11. Cory-Slechta, D.A.; Virgolini, M.B.; Thiruchelvam, M.; Weston, D.D.; Bauter, M.R. Maternal stress modulates the effects of developmental lead exposure. Environ. Health Persp. 2004, 112, 717-730.

12. Rossi-George, A.; Virgolini, M.; Weston, D.; Thiruchelvam, M.; Cory-Slechta, D. Interactions of lifetime lead exposure and stress: Behavioral, neurochemical and HPA axis effects. Neurotoxicology 2011, 32, 83-99.

13. Rabinowitz, M.B.; Wetherill, G.; Kopple, J.D. Kinetic analysis of lead metabolism in healthy humans. J. Clin. Invest. 1976, 58, 260-270.

14. Smith, J.; Farris, F. Methyl mercury pharmacokinetics in man: A reevaluation. Toxicol. Appl. Pharmacol. 1996, 137, 245-252.

15. Hellhammer, D.; Stone, A.; Hellhammer, J.; Broderick, J. Measuring Stress. In Encyclopedia of Behavioural Neuroscience; Koob, G., Moal, M.L., Thompson, R., Eds.; Academic Press: Oxford, UK, 2010; pp. 186-191.

16. McEwen, B.S. Protective and damaging effects of stress mediators. N. Engl. J. Med. 1998, 338, 171-179. 
17. Sterling, P.; Eyer, J. Allostasis: A New Paradigm to Explain Arousal Pathology. In Handbook of Life Stress, Cognition and Health; Fisher, S., Reason, J., Eds.; Wiley: New York, NY, USA, 1988; pp. 629-649.

18. Centers for Disease Control and Prevention (CDC). National Health and Nutrition Examination Survey: Public Data General Release File Documentation; CDC National Center for Health Statistics: Atlanta, GA, USA, 2006.

19. Centers for Disease Control and Prevention (CDC). Laboratory Procedure Manual: Lead, Cadmium, and Mercury in Whole Blood; CDC National Center for Environmental Health: Atlanta, GA, USA, 2004.

20. U.S. Environmental Protection Agency. Supplementary Guidance for Conducting Health Risk Assessment of Chemical Mixtures; EPA: Washington, DC, USA, 2000.

21. Mahaffey, K.R.; Clickner, R.P.; Bodurow, C.C. Blood organic mercury and dietary mercury intake: National Health and Nutrition Examination Survey, 1999 and 2000. Environ. Health Persp. 2004, 112, 562-570.

22. U.S. Environmental Protection Agency (EPA). Methylmercury (MeHg) (CASRN 22967-92-6). In Integrated Risk Information System (IRIS); EPA: Washington, DC, USA, 2001.

23. Jedrychowski, W.; Perera, F.; Jankowski, J.; Mrozek-Budzyn, D.; Mroz, E.; Flak, E.; Edwards, S.; Skarupa, A.; Lisowska-Miszczyk, I. Gender specific differences in neurodevelopmental effects of prenatal exposure to very low-lead levels: The prospective cohort study in three-year olds. Early Hum. Dev. 2009, 85, 503-510.

24. Amaral, J.H.; Rezende, V.B.; Quintana, S.M.; Gerlach, R.F.; Barbosa, F.; Tanus-Santos, J.E. The relationship between blood and serum lead levels in peripartum women and their respective umbilical cords. Basic Clin. Pharmacol. Toxicol. 2010, 107, 971-975.

25. Carlisle, J.C.; Dowling, K.C.; Siegel, D.M.; Alexeeff, G.V. A blood lead benchmark for assessing risks from childhood lead exposure. J. Environ. Sci. Health. A 2009, 44, 1200-1208.

26. Centers for Disease Control and Prevention (CDC). What Do Parents Need to Know to Protect. Their Children? CDC: Atlanta, GA, USA, 2012.

27. Juster, R.P.; McEwen, B.S.; Lupien, S.J. Allostatic load biomarkers of chronic stress and impact on health and cognition. Neurosci. Biobehav. Rev. 2010, 35, 2-16.

28. Chyu, L.; Upchurch, D.M. Racial and ethnic patterns of allostatic load among adult women in the United States: Findings from the National Health and Nutrition Examination Survey 1999-2004. J. Womens Health 2011, 20, 575-583.

29. Crimmins, E.M.; Johnston, M.; Hayward, M.; Seeman, T. Age differences in allostatic load: An index of physiological dysregulation. Exp. Gerontol. 2003, 38, 731-734.

30. Hawkley, L.C.; Lavelle, L.A.; Berntson, G.G.; Cacioppo, J.T. Mediators of the relationship between socioeconomic status and allostatic load in the Chicago Health, Aging, and Social Relations Study (CHASRS). Psychophysiology 2011, 48, 1134-1145.

31. Axelrad, D.A.; Cohen, J. Calculating summary statistics for population chemical biomonitoring in women of childbearing age with adjustment for age-specific natality. Environ. Res. 2011, 111, $149-155$. 
32. Fortin, M.C.; Cory-Slechta, D.A.; Ohman-Strickland, P.; Nwankwo, C.; Yanger, T.S.; Todd, A.C.; Moynihan, J.; Walton, J.; Brooks, A.; Fiedler, N. Increased lead biomarker levels are associated with changes in hormonal response to stress in occupationally exposed male participants. Environ. Health Persp. 2012, 120, 278-283.

33. Geer, L.A.; Persad, M.D.; Palmer, C.D.; Steuerwald, A.J.; Dalloul, M.; Abulafia, O.; Parsons, P.J. Assessment of prenatal mercury exposure in a predominately Caribbean immigrant community in Brooklyn, NY. J. Environ. Monit. 2012, 14, 1035-1043.

34. Agency for Toxic Substances and Disease Registry (ATSDR). Interaction Profile for: Chlorpyrifos, Lead, Mercury, and Methylmercury; ATSDR: Atlanta, GA, USA, 2006.

35. Aylward, L.L.; Kirman, C.R.; Schoeny, R.; Portier, C.J.; Hays, S.M. Evaluation of biomonitoring data from the CDC national exposure report in a risk assessment context: Perspectives across chemicals. Environ. Health Persp. 2013, 121, 287-294.

36. U.S. Environmental Protection Agency (EPA). Integrated Risk Information System (IRIS); EPA: Washington, DC, USA, 2013.

37. DeFur, P.L.; Evans, G.W.; Cohen Hubal, E.A.; Kyle, A.D.; Morello-Frosch, R.A.; Williams, D.R. Vulnerability as a function of individual and group resources in cumulative risk assessment. Environ. Health Persp. 2007, 115, 817-824.

38. Nelson, J.W.; Scammell, M.K.; Hatch, E.E.; Webster, T.F. Social disparities in exposures to bisphenol A and polyfluoroalkyl chemicals: A cross-sectional study within NHANES 2003-2006. Environ. Health 2012, 11, 1-15.

39. Crimmins, E.M.; Kim, J.K.; Alley, D.E.; Karlamangla, A.; Seeman, T. Hispanic paradox in biological risk profiles. Am. J. Public Health 2007, 97, 1305-1310.

40. Martin, J.; Hamilton, B.; Sutton, P.; Ventura, S.; Menacker, F.; Munson, M. Births: Final Data for 2003; National Center for Health Statistics: Hyattsville, MD, USA, 2005.

41. Hertz-Picciotto, I.; Schramm, M.; Watt-Morse, M.; Chantala, K.; Anderson, J.; Osterloh, J. Patterns and determinants of blood lead during pregnancy. Am. J. Epidemiol. 2000, 152, 829-837.

42. Woodruff, T.J.; Zota, A.R.; Schwartz, J.M. Environmental chemicals in pregnant women in the United States: NHANES 2003-2004. Environ. Health Persp. 2011, 119, 878-885.

43. Kudielka, B.M.; Wust, S. Human models in acute and chronic stress: assessing determinants of individual hypothalamus-pituitary-adrenal axis activity and reactivity. Stress 2010, 13, 1-14.

44. Morello-Frosch, R.; Shenassa, E.D. The environmental "riskscape" and social inequality: Implications for explaining maternal and child health disparities. Environ. Health Persp. 2006, $114,1150-1153$.

45. Morrison, S.; Shenassa, E.D.; Mendola, P.; Wu, T.; Schoendorf, K. Allostatic load may not be associated with chronic stress in pregnant women, NHANES 1999-2006. Ann. Epidemiol. 2013, 23, 294-297.

46. Wallace, M.E.; Harville, E.W. Allostatic load and birth outcomes among white and black women in New Orleans. Matern Child Health J. 2013, 17, 1025-1029.

47. Clougherty, J.E.; Levy, J.I.; Kubzansky, L.D.; Ryan, P.B.; Suglia, S.F.; Canner, M.J.; Wright, R.J. Synergistic effects of traffic-related air pollution and exposure to violence on urban asthma etiology. Environ. Health Persp. 2007, 115, 1140-1146. 
48. Chen, E.; Schreier, H.M.; Strunk, R.C.; Brauer, M. Chronic traffic-related air pollution and stress interact to predict biologic and clinical outcomes in asthma. Environ. Health Persp. 2008, 116, 970-975.

49. Shankardass, K.; McConnell, R.; Jerrett, M.; Milam, J.; Richardson, J.; Berhane, K. Parental stress increases the effect of traffic-related air pollution on vhildhood ssthma incidence. Proc. Natl. Acad. Sci. USA. 2009, 106, 12406-12411.

50. Hicken, M.T.; Gee, G.C.; Connell, C.; Snow, R.C.; Morenoff, J.; Hu, H. Black-white blood pressure disparities: Depressive symptoms and differential vulnerability to blood lead. Environ. Health Persp. 2013, 121, 205-209.

51. Read, S.; Grundy, E. Allostatic Load-A Challenge to Measure Multisystem Physiological Dysregulation. Pathways Node at NCRM; National Centre for Research Methods: Oxford, UK, 2012.

52. Seplaki, C.L.; Goldman, N.; Glei, D.; Weinstein, M. A comparative analysis of measurement approaches for physiological dysregulation in an older population. Exp. Gerontol. 2005, 40, 438-449.

(C) 2014 by the authors; licensee MDPI, Basel, Switzerland. This article is an open access article distributed under the terms and conditions of the Creative Commons Attribution license (http://creativecommons.org/licenses/by/3.0/). 